\title{
Originalartikel
}

\section{Zelluläre Abwehrmechanismen in der Hämolymphe und der Nachweis spezifischer Zelltypen im Seminalplasma bei Apis mellifera $\mathrm{L}$}

\author{
S Mitro \\ Zentrum für kontinentale Agrar- und Wirtschaftsforschung der Justus-Liebig Universität Gießen, \\ Otto-Behaghel Str 10, D-35394 Gießen, Deutschland
}

(Eingegangen 20 Mai 1993; Angenommen 10 Dezember 1993)

\begin{abstract}
Zusammenfassung - Mikroskopische Untersuchungen der Bienenhämolymphe nach Inkubation mit Hühner- bzw Schweineerytrozyten zeigen, daß bestimmte Zelltypen (granuläre Zellen und Plasmatozyten) in der Lage sind, die als fremd erkannten Zellen mit Hilfe von Zellschleiern, Zellausläufern oder Scheinfüßchen anzugreifen und zu verändern. Die in der Hämolymphe vorkommenden granulären Zellen und Plasmatozyten wurden auch in Ausstrichpräparaten des Seminalplasmas von Drohnen nachgewiesen. Daneben wurden im Seminalplasma Riesenzellen und spindelförmige Zellen beobachtet, die in der Hämolymphe nicht nachzuweisen waren.
\end{abstract}

Apis mellifera / Abwehrmechanismen / Hämozyten / Seminalplasma

\section{EINLEITUNG}

Bei Insekten in der Hämolymphe vorkommende Hämozyten sind wichtige Abwehrfaktoren gegenüber schädlichen Substanzen. Ihnen wird nach Anheften an fremde Substanzen die Ausschüttung chemotaktisch wirksamer Substanzen zugeschrieben, die zelluläre Abwehrvorgänge vermitteln und einleiten. Bisher wurden bei verschiedenen Insekten vielfältige Hämozyten-Typen beschrieben (Götz und Boman, 1985; Dunn, 1986; Boman und Hultmark, 1987; Lackie, 1988). Zum Grundmuster der Insektenzellen gehören nach bisherigen
Erkenntnissen undifferenzierte Prohämozyten, phagozytosefähige Plasmatozyten und granuläre Zellen, Oenocyten, Adipohämozyten und Koagulozyten. Immunologische Fragestellungen bei Bienen setzen Kenntnisse über das Vorkommen und die Bedeutung von Zellen voraus.

Über Zellen in der Bienen-Hämolymphe berichten Wille (1973) und Wille und Vecchi (1974). Nach Kontakt mit körperfremden Substanzen (Varroa-Parasitierung) können im Blutbild Veränderungen auftreten (Glinski und Jarosz, 1988). Da neben der Hämolymphe weitere Organsysteme der Biene (Darmtrakt, Genitalorgane) einer dauern- 
den Gefahr der Schädigung durch fremde Substanzen ausgesetzt sind, übernehmen vermutlich auch hier bestimmte Zellen bzw andere aktive Substanzen wichtige Schutzfunktionen. Qualitative oder quantitative Veränderungen des physiologischen Zellbildes in der Hämolymphe bzw in anderen Körperflüssigkeiten können Hinweise auf den Gesundheitszustand des untersuchten Organismus geben.

In der Arbeit wird über vorläufige Untersuchungen an Zellelementen der Bienenhämolymphe und des Seminalplasmas von Drohnen berichtet. Mit Hilfe von Agglutinationsversuchen wurde das Verhalten der Bienenhämozyten gegenüber Erytrozyten vom Huhn und Schwein getestet. Weiterhin wurden Seminalplasma-Ausstriche von Drohnen auf das Vorkommen bestimmter Zellen untersucht.

\section{MATERIAL UND METHODEN}

\section{Probenentnahme}

Die Hämolymphe-Entnahme erfolgte aus dem Abdomen der Bienen. Dabei wurde mittels einer Spritze dorsal in den Intersegmentalbereich zwischen den zweiten und dritten Tergiten eingestochen.

Die Gewinnung des Seminalplasmas erfolgte nach Zentrifugation der Spermaproben bei 2000 $\mathrm{U} / \mathrm{min}$ während einer Zeit von $20 \mathrm{~min}$.

\section{Färbung}

Die Bienenhämolymphe und das Seminalplasma wurden in einer Menge von je $2 \mu$ auf Objektträgern ausgestrichen und nach Pappenheim gefärbt.

\section{Agglutinationsversuche}

Frisch gewonnene Hämolymphe wurde mit gewaschenen Erytrozyten (Ec) von Huhn bzw Schwein bei Zimmertemperatur 7 Stunden inkubiert. Die Überprüfung der Agglutinationsfähigkeit der Erytrozyten erfolgte durch Verdünnungsreihen der Hämolymphe mit 0,9\%iger Kochsalzlösung.

Die Versuchsansätze zur Überprüfung der Agglutinationsfähigkeit sowie die Ausstriche des Seminalplasmas von Drohnen wurden im Lichtmikroskop bei tausendfacher Vergrößerung ausgewertet.

\section{ERGEBNISSE}

Eine Lysis der im Agglutinationsversuch eingesetzten Erytrozyten durch Bestandteile der Hämolymphe wurde nach bisherigen Ergebnissen nicht festgestellt.

Die panoptische Färbung nach Pappenheim ist für die Darstellung von Bienenzellen in der Hämolymphe und im Seminalplasma geeignet.

Bei der mikroskopischen Auswertung der durchgeführten Agglutinationsversuche der Hämolymphe mit Erytrozyten von Huhn und Schwein werden zwei Hämozytentypen beobachtet, die den bei anderen Insekten beschriebenen phagozytosefähigen Plasmatozyten und granulären Zellen entsprechen.

Diese Zellen der Bienenhämolymphe sind in der Lage, Huhn und SchweineErytrozyten als fremd zu erkennen. Die Hämozyten besitzen die Fähigkeit, die Fremdzellen mit Hilfe von Zellschleiern, Zellausläufern oder Scheinfüßchen anzugreifen und zu verändern.

Die Plasmatozyten und die granulären Zellen haben zahlreiche Einschlüsse in inrem Zytoplasma (Abb 1-3).

Die in der Hämolymphe vorkommenden granulären Zellen und Plasmatozyten wurden auch in mehreren untersuchten Ausstrichen des Seminalplasmas von Drohnen nachgewiesen. Im Vergleich zur Hämolymphe kommen die granulären Zellen häufiger als die Plasmatozyten vor. Riesenzel- 


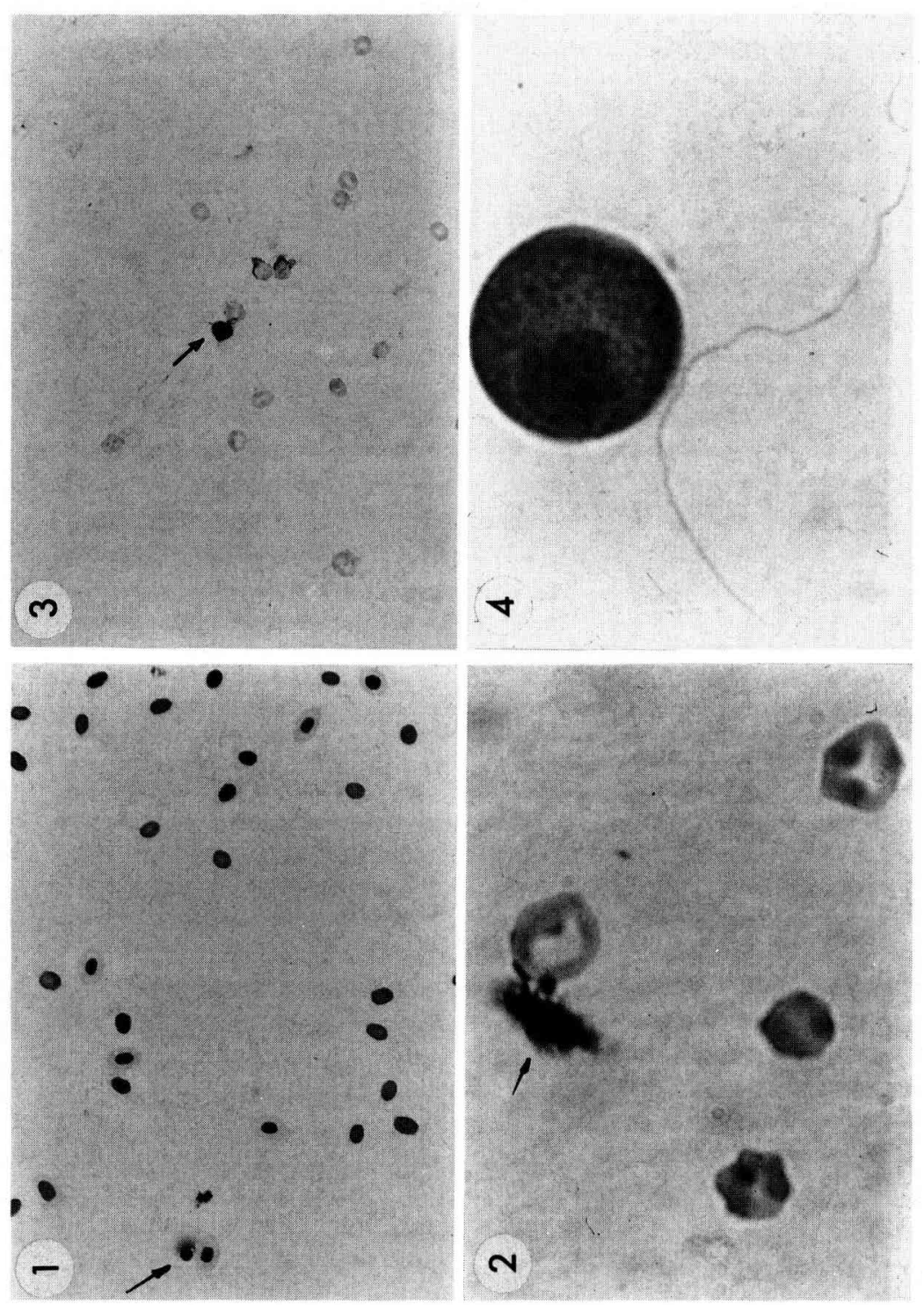

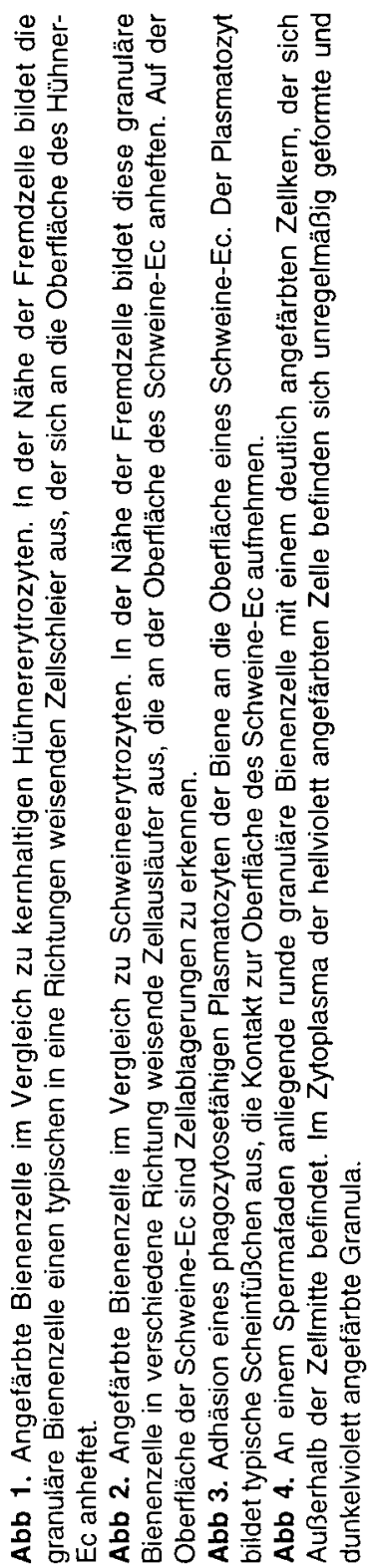



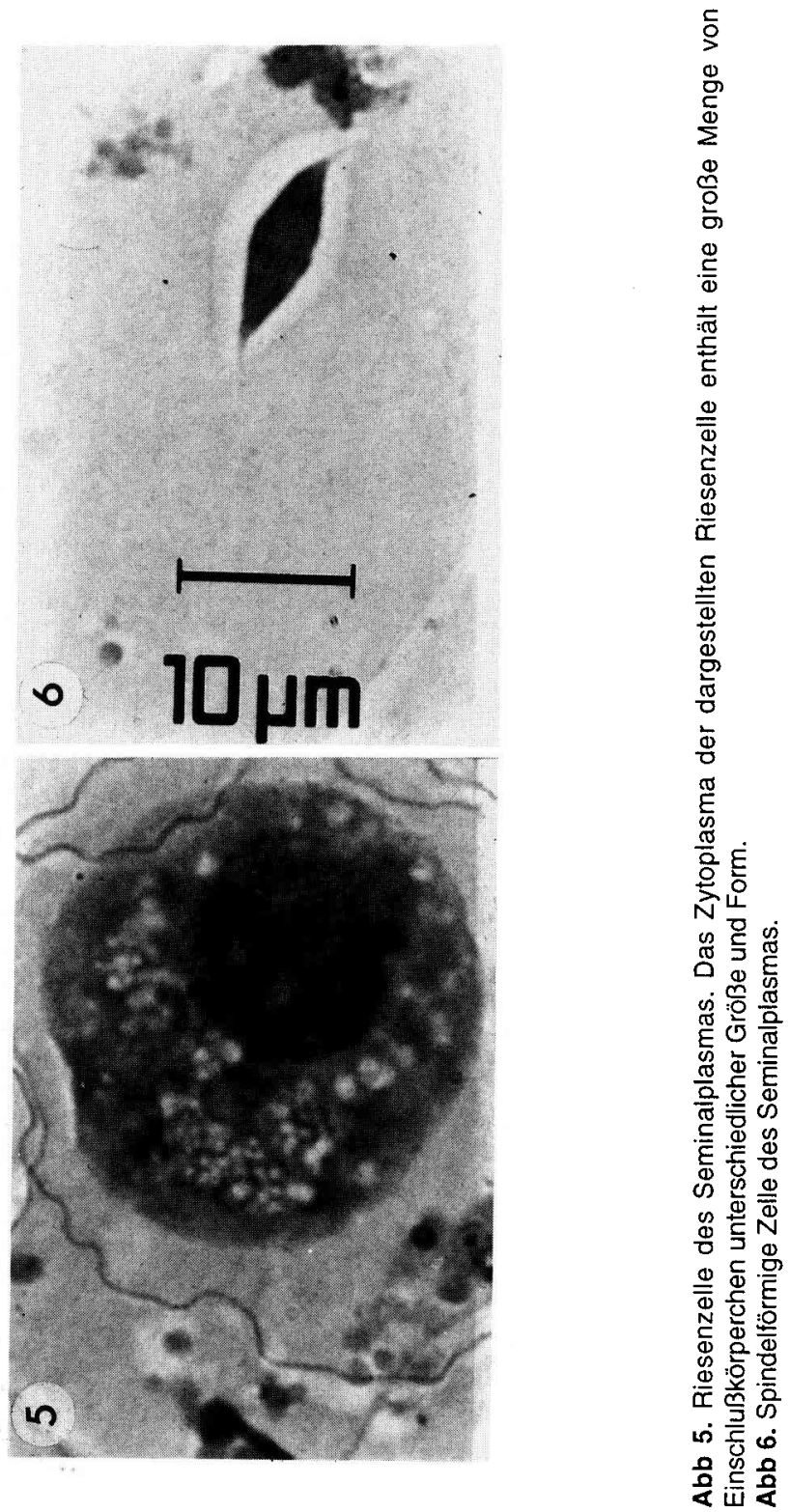
len und spindelförmige Zellen wurden dagegen nur im Seminalplasma nachgewiesen (Abb 4-6).

\section{DISKUSSION}

Es kann eingeschätzt werden, daß gefärbte Hämolymphe-Ausstriche in Hinsicht auf das Vorkommen einzelner Zelltypen nicht mit den Seminalplasma-Ausstrichen gleichgesetzt werden können. Aus den ersten Untersuchungen geht weiter hervor, daß in das Seminalplasma Zellen gelangen können, die den granulären Zellen und Plasmatozyten der Hämolymphe entsprechen.

In der Hämolymphe übernehmen solche Zellen wichtige Abwehrfunktionen. Für andere Arthropoden sind granuläre Zellen und Plasmatozyten als wichtige Phagozyten beschrieben worden (Rahmet-Alla und Rowley, 1990). Das Vorkommen der granulären Zellen und Plasmatozyten im Seminalplasma deutet darauf hin, daß auch in Körpersekreten durch Zellen vermittelte lokale Abwehrvorgänge eine Rolle spielen könnten.

Daneben wurden im Seminalplasma sogenannte Riesenzellen und spindelförmige Zellen beobachtet, die in der Hämolymphe nicht vorkamen. Über die Herkunft und Bedeutung dieser Zellen können nach bisherigen Untersuchungen keine Aussagen getroffen werden. Gegenstand weiterer Untersuchungen wird zunächst die exakte qualitative und quantitative Bewertung einzelner Zellelemente im Blut und in den Körpersekreten der Biene sein.

\footnotetext{
Résumé - Mécanismes cellulaires de défense de l'hémolymphe et description de cellules spécifiques dans le plasma séminal d'Apis mellifera $L$. On a mis à incuber de l'hémolymphe d'abeille avec des érythrocytes de poule et de porc. Après coloration selon Pappenheim, l'analyse en
}

microscopie optique a montré 2 types d'hémocytes présentant des réactions de défense: des cellules granulaires et des plasmocytes, qui ont réagi aux cellules étrangères par des adhésions cellulaires comme les pseudopodes ou les voiles cellulaires (figs 1-3). On a également trouvé des cellules granulaires et des plasmocytes dans le plasma séminal des mâles (fig 4), ainsi que d'autres cellules géantes et des cellules fusiformes, qui n'étaient pas présentes dans l'hémolymphe. La présence de ces diverses cellules dans le plasma séminal indique qu'il existe au niveau cellulaire local des réactions de défense.

réaction de défense / hémocyte / plasma séminal / réaction immune

\begin{abstract}
Summary - Cellular defence reaction of the hemolymph and description of specific cells in the seminal plasma of Apis mellifera L. Hemolymph of honey bees was incubated with erythrocytes of hens and pigs. After staining according to Pappenheim, microscopic analysis revealed two types of hemocytes with defence reactions: granular cells and plasmocytes reacted with foreign cells with cellular adhesions such as pseudopodia or cellular veils (figs 1-3). Granular cells and plasmocytes were also found in the seminal plasma (fig 4). Further giant cells and spindle shaped cells were found which were not present in the hemolymph. The occurrence of various cells in the seminal plasma indicates the presence of local cellular defence reactions there.
\end{abstract}

\section{Apis mellifera / defence reactions / hemo- cytes / seminal plasma}

\section{LITERATUR}

Boman HG, Hultmark D (1987) Cell-free immunity in insects. Annu Rev Microbiol 41, 103-126 
Dunn PE (1986) Biochemical aspects of insect immunology. Annu Rev Entomo/31, 321-339

Glinski Z, Jarosz J (1988) Varroa jacobsoni invasion and the level of cell-free immunity in upright larvae of the worker honey bee, Apis melifera. Folia Vet 32 , 39-50

Götz P, Boman HG (1985) Insect Immunity. In: Comprehensive Insect Physiology, Biochemistry and Pharmacology, Vol 3 (Kerkut GA, Gilbert LI, eds) Pergamon, New York, 453-485

Lackie AM (1988) Immune mechanisms in insects. Parasitol Today 4, 98-105
Rahmet-Alla M, Rowley AF (1990) Studies on the cellular defence reactions of the Madeira cockroach, Leucophaea maderae: In vitro phagocytosis of different strains of Bacillus cereus and their effect on hemocyte viability. J Invertebr Pathol 55, 350-356

Wille $H$ (1973) Beziehungen zwischen Überlebensdauer, Krankheitsbefunden und dem Blutbild erwachsener Bienen (Apis mellifica L). Schweiz Landwirtsch Forsch 12, 269-289

Wille $H$, Vecchi MA (1974) Untersuchungen über die Hämolymphe der Honigbiene (Apis mellifica L). Mitt Schweiz Entomol Ges 47, 133-149 